

\title{
Adaptive Delta Modulation in Networked Controlled Systems With bounded Disturbances
}

\author{
Fabio Gomez-Estern, Carlos Canudas de Wit, Francisco Rubio
}

\section{To cite this version:}

Fabio Gomez-Estern, Carlos Canudas de Wit, Francisco Rubio. Adaptive Delta Modulation in Networked Controlled Systems With bounded Disturbances. IEEE Transactions on Automatic Control, 2011, 56 (1), pp.129-134. 10.1109/TAC.2010.2083370 . hal-00582444

HAL Id: hal-00582444

https://hal.science/hal-00582444

Submitted on 1 Apr 2011

HAL is a multi-disciplinary open access archive for the deposit and dissemination of scientific research documents, whether they are published or not. The documents may come from teaching and research institutions in France or abroad, or from public or private research centers.
L'archive ouverte pluridisciplinaire HAL, est destinée au dépôt et à la diffusion de documents scientifiques de niveau recherche, publiés ou non, émanant des établissements d'enseignement et de recherche français ou étrangers, des laboratoires publics ou privés. 


\title{
Adaptive Delta Modulation in Networked Controlled Systems with bounded disturbances
}

\author{
Fabio Gómez-Estern, Carlos Canudas-de-Wit, and Francisco R. Rubio
}

\begin{abstract}
This paper investigates the closed-loop properties of the differential coding scheme known as Delta Modulation $(\Delta-M)$ when used in feedback loops within the context of linear systems controlled through a communication network. We propose a new adaptive scheme with variable quantization step $\Delta$, by defining an adaptation law exclusively in terms of information available at both the transmitter and receiver. With this approach, global asymptotic stability of the networked control system is achieved for a class of controllable (possible unstable) linear plants. Moreover, thanks to the globally defined switching policy, this architecture enjoys a disturbance rejection property that allows the system to recover from any finite-time unbounded disturbance or communication loss.
\end{abstract}

\section{Index Terms}

Differential Coding, Delta Modulation, stabilization of Networked Control Systems.

\section{INTRODUCTION}

D ELTA Modulation $(\Delta-M)$ is a well-known differential coding technique used for reducing the data rate required for voice communication, see [1]. The standard technique is based on synchronizing a state predictor on emitter and receiver and just sending a one-bit error signal corresponding to the innovation of the sampled data with respect to the predictor. The prediction is then updated by adding a positive or negative quantity (determined by the bit that has been transmitted) of absolute value $\Delta$, a known parameter shared between emitter and receiver. Hence,

C. Canudas-de-Wit is with The Laboratoire d'Automatique de Grenoble, UMR CNRS 5528, Grenoble, FRANCE. carlos.canudas-de-witegipsa-lab.inpg.fr.

Fabio Gómez-Estern, Francisco R. Rubio are with the Department of Automatic Control and Systems Engineering at the University of Sevilla, Spain. Email: fabiodesi.us.es, rubiodesi.us.es, 
$\Delta$ can be regarded as the quantization step. This paper proposes an adaptive extension a of fixedgain differential coding scheme previously introduced by the same authors (see [2]) in the context of linear systems interconnected through some transmission network.

The selection of $\Delta$ is a crucial issue on the quality of the decoded signals. It is well known in digital communications framework that large values of $\Delta$ will result in a high granular noise, while too small values of $\Delta$ will result in slope-overload distortion. In closed-loop configurations, as in the scenarios considered here, the choice of $\Delta$ is even more important because it may cause instability. The closed-loop stability properties of Delta Modulation coding with fixed or scheduled gains, has been studied in [2], [3], [4].

Delta modulation $(\Delta-M)$ algorithm can also be understood as the coarsest two-level (1bit) quantizer. Thus, this technique is a simple alternative to approaches concerning the use of quantizers in the context of NCS, i.e. [5], [6], [7], [8], [9], [10], [11] among others.

For a Delta Modulation scheme with constant quantization step $\Delta$ it was shown in [2] that only a limited domain of attraction was obtained. In addition, the state was only guaranteed to converge asymptotically to a finite ball, being its size related to the parameters of the open-loop plant, and to the user-defined parameter $\Delta$.

By making $\Delta$ an adaptive quantity, more effective schemes of $\Delta$-modulation have been already proposed in the communications community [1]. The idea is to design an update law for $\Delta$, defined exclusively in terms of the information available both at the receiver and transmitter, aiming at improving the resolution of the differential coding by reducing the gain $\Delta$ for slowly varying signals, while enlarging $\Delta$ in case of rapid change of the input, and hence allowing for faster signal tracking and higher bandwidth of the transmitted signals. So far in the communications field, adaptation laws for $\Delta$ have been proposed under somewhat heuristic criteria, as little information is supposed to be available on the dynamics of the signal source. However, when dealing with feedback systems, the dynamical properties of the plant become very useful in designing the adaptive law. This problem, to which this paper is devoted. is framed as shown in Figure 1.

The main paper contribution is the introduction of an adaptation mechanism consisting of varying the quantization interval $\Delta$ in terms of a minimal amount of information available at the transmitter and the receiver. This type of adaptation law, although well known in the communications field, is used and analyzed for the first time a feedback configuration shown 


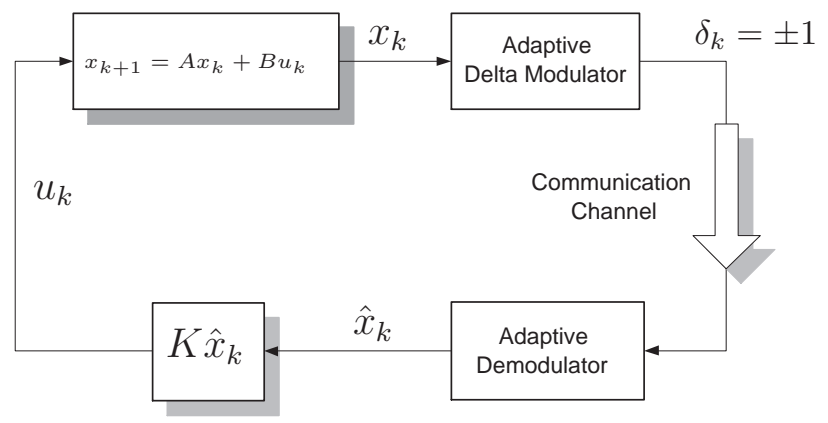

Fig. 1. Block diagram of the problem set up studied in this paper.

in Figure 1. It is also shown that this adaptive coding structure, modified as proposed in [2], is proven to yield closed-loop global asymptotic stability for a class of open-loop unstable linear systems. We provide also a comparison between our approach and existing ones, in order highlight the main advantage: a disturbance recovery property that guarantees that if the system state is driven temporarily away from the origin, due to some unattended unbounded finite-time disturbance, or possibly loss of communication, the system will ultimately stabilize the estimation error, and drive the state back to the origin. This is due to the globally defined switching policy (and not only in one time sense as happens in most of the referenced papers), and also allows a free initialization of the encoder without any previous information of the state.

The results are presented in scalar form. The extensions to higher dimension are easy to develop in the case of diagonalizable system matrices, and will be spared for the sake of space. However, for non-diagonalizable systems, the analysis is involved, and the technical details have been presented in [12] for the fixed Delta Modulation algorithm, while the adaptive version is part of an ongoing research work.

\section{AdAptive $\Delta-M$ CODing SCheme}

The problem setup will be initially presented, and the fixed-step Delta Modulation networked controller [2] will be briefly recalled for the sake of completeness. Then we propose a $\Delta-$ adaptation law resulting in a global asymptotic convergence of the state estimation error and system states to zero. This is a significant achievement with respect to the fixed-gain scheme presented in [2]. 


\section{A. Assumptions}

In the following, we will assume that the transmitted information is binary $\delta_{k} \in\{-1,1\}$, that only sensor-to-controller transmission is allowed, that a $100 \%$ reliable noiseless channel without transmission delays is used, and that the data is sent at a fixed rate (we select the sampling frequency in order to transmit only one $\delta_{k}$ at a time).

\section{B. Problem Setup}

Consider first the following one-dimensional discrete time-system, together with the control law, and the fixed-step differential coding law:

\begin{tabular}{|c|c|}
\hline Open-loop system, and encoder: & Control law and decoder: \\
\hline$x_{k+1}=a x_{k}+b u_{k}$ & $\hat{x}_{k+1}=[a-b K] \hat{x}_{k}+\Delta \cdot \delta_{k}$ \\
\hline$\hat{x}_{k+1}=[a-b K] \hat{x}_{k}+\Delta \cdot \delta_{k}$ & $u_{k}=-K \hat{x}_{k}$ \\
\hline$\hat{x}_{0}=0, \delta_{k} \triangleq \operatorname{sgn}\left(x_{k}-\hat{x}_{k}\right)$ & $\hat{x}_{0}=0$ \\
\hline
\end{tabular}

In that structure, $x \in \mathbb{R}$ is the state of the plant, and a standard Delta Modulator is used to encode it. This structure uses a prediction of the state $\hat{x}_{k}$ generated by a model of the closedloop dynamics (2) synchronized at both communication ends. When a new sample of the state $x_{k}$ arrives at the encoder, it is compared to the prediction $\hat{x}_{k}$, and its difference is coded on a binary basis, i.e. the value of $\operatorname{sgn}\left(x_{k}-\hat{x}_{k}\right)$ is coded and transmitted as a binary digit.

Stability of this system has been analyzed in [2]. Although it has interesting disturbance rejection properties, the main limitation of this approach is the fact that stability is semiglobal, in the sense that the quantization step must be chosen as a function of an upper bound of the initial state. This is a quite frequent fact in the literature (see [7]), and has been tackled (in that paper) by devising open-loop initialization mechanisms for estimating that bound. Another issue (also shown in [2]) is that using fixed-step Delta Modulation, the state does not converge strictly to zero, but to a finite ball around the origin, whose radius is proportional to $\Delta$ (there is chattering in steady state). As a consequence, when the gain $\Delta$ is fixed, there is an inherent trade-off between stability and precision. This motivates our search for other coding strategies with variant gains, as shown next. 


\section{Adaptation law design}

Adaptive $\Delta$-modulation in Digital Communications aims at improving the resolution of the differential coding scheme according to the size of the signals to be transmitted. In our case this signal is the system state, hence a reasonable approach is to enlarge $\Delta$ for large values of the estimated states, and decrease it for smaller values. Another requisite is the Equi-Memory property described in [13], which suggests that the quantization step must be the same at the transmitter and the receiver at any time. Hence, the adaptation law must be defined exclusively in terms of the shared information, i.e. of $\left\{\delta_{0}, \delta_{1}, \ldots, \delta_{k}\right\}$. Another condition is to keep the adaptation law as simple as possible, and also minimize its memory usage. With that aim we will propose a very heuristic and simple approach, and will further provide a detailed analysis to prove that it guarantees global asymptotic stability. In order to design an $\Delta$-adaptive mechanism to achieve global asymptotic stability two opposite behaviors must be observed in the analysis of [2]. For large values of the estimation error $\tilde{x}$, there is probably slope overload, so $\Delta_{k}$ should grow at a higher rate than the plant escape velocity. When the state is trapped into a domain of attraction for the present $\Delta_{k}$, the step size must decrease (for improving resolution) at a slower rate than the state convergence in order to prevent it from getting too small relative to the state.

From this intuition, an adaptive scheme with minimal storage and computation requirements is proposed as follows:

1) If $\delta_{k}=\delta_{k-1}$ then the state is assumed to be escaping, thus $\Delta_{k}$ must be increased.

2) If $\delta_{k} \neq \delta_{k-1}$ then the state is assumed to converge (oscillations close to zero) and $\Delta_{k}$ must be decreased.

The following update law is proposed:

$$
\begin{aligned}
\Delta_{k+1} & =\phi_{k+1} \Delta_{k} \\
\phi_{k+1} & =\left\{\begin{array}{l}
\lambda^{+} \text {if } \delta_{k}=\delta_{k-1} \\
\lambda^{-} \text {if } \delta_{k} \neq \delta_{k-1}
\end{array}\right.
\end{aligned}
$$

where $0<\lambda^{-}<1$ is the exponential decay rate of $\Delta_{k}$, and $\lambda^{+}>1$ is the exponential growth rate. The proposed algorithm is shown in Fig.2. This adaptation law can be seen as a generalization of Jayant's adaptation rule (1970) (see [1]). 


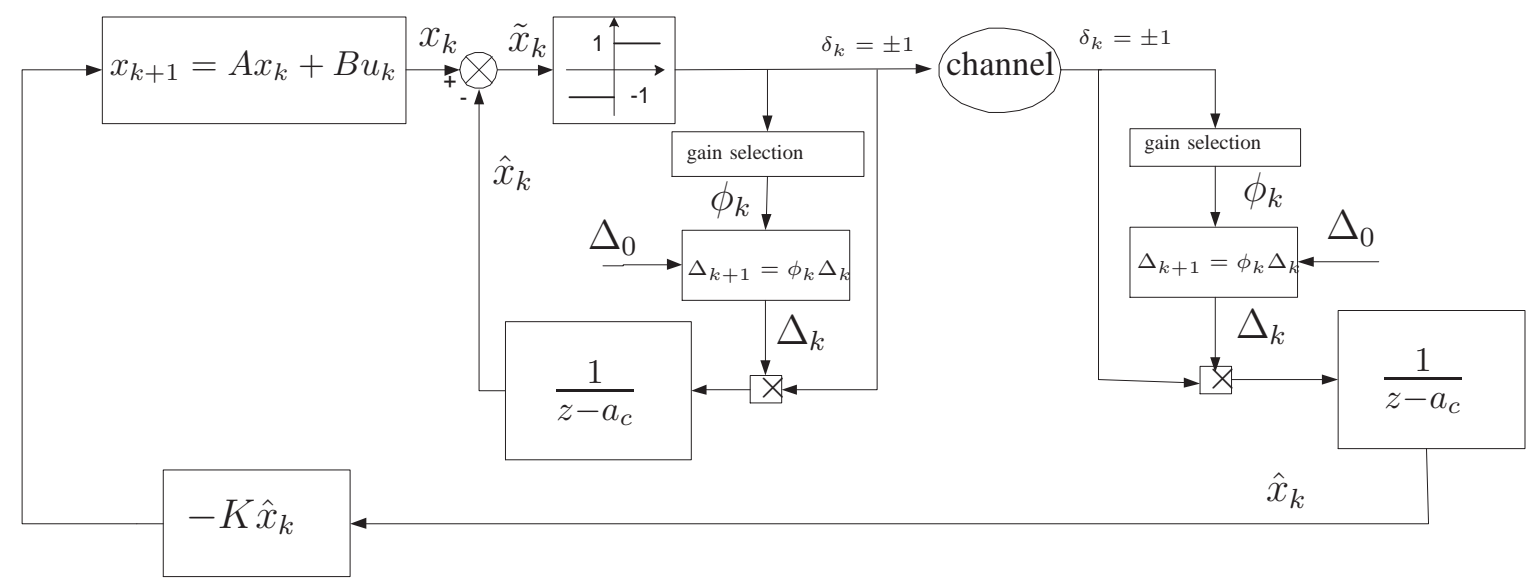

Fig. 2. Adaptive coding scheme. The figure shows the case of one-dimensional systems. The selection gain block toggles the value of $\phi_{k}$ according to Equation (6).

\section{Error equations}

The complete feedback system with the adaptive delta Delta Modulation coding scheme is then:

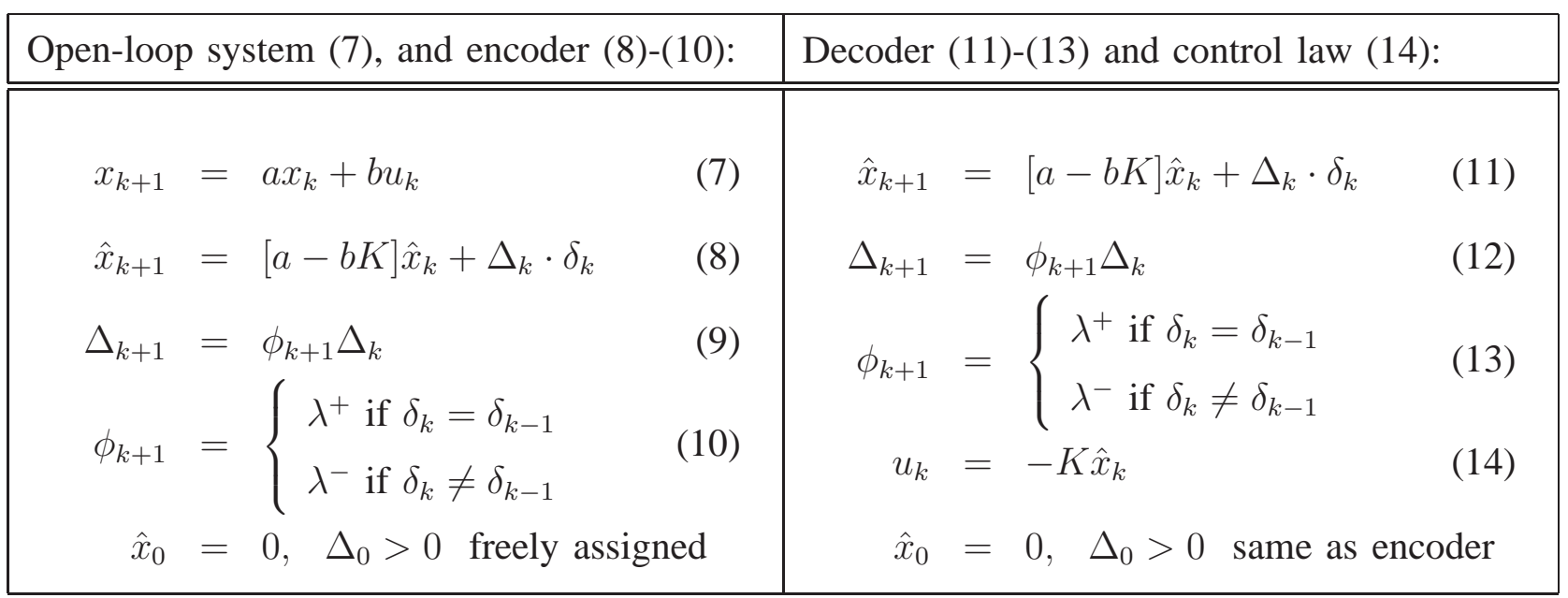

With the above definitions, the closed-loop error dynamics become

$$
\begin{aligned}
\tilde{x}_{k+1} & =a \tilde{x}_{k}-\Delta_{k} \cdot \delta_{k} \\
\Delta_{k+1} & =\phi_{k+1} \Delta_{k}, \quad \Delta_{0}>0 \\
\phi_{k+1} & =\lambda^{-}+\frac{1}{2}\left(\lambda^{+}-\lambda^{-}\right)\left|\delta_{k+1}+\delta_{k}\right|
\end{aligned}
$$

The causality of the system is guaranteed because the computation of $\tilde{x}_{k+1}$ is only based on $\tilde{x}_{k}$ 
and older values. The following Theorem states the stability of the closed-loop system.

\section{E. Main result (stability analysis)}

Theorem 1: The error trajectories $\tilde{x}_{k}$ of system (15), resulting from the adaptive $\Delta$-modulation coding scheme (7)-(14), globally asymptotically converge to zero as $k \rightarrow \infty$ if there exist parameters $\lambda^{+}>1, \lambda^{-} \in(0,1)$ satisfying the following inequalities:

$$
\begin{aligned}
& \lambda^{+}>a \\
& \lambda^{-}<\left(\lambda^{+}\right)^{-\frac{\beta}{2}},
\end{aligned}
$$

$$
\text { where } \quad \beta\left(a, \lambda^{-}, \lambda^{+}\right) \triangleq 1+\log _{\rho}\left(1+\frac{a\left(a-\lambda^{-}\right)(\rho-1)}{\left(\lambda^{-}\right)^{2}}\right) \quad \text { and } \quad \rho \triangleq \frac{\lambda^{+}}{a}
$$

Moreover, $\Delta_{k}$ and hence $x_{k}$ also converge to zero regardless the initial conditions $\left(x_{0}, \Delta_{0}\right)$.

Proof: The claim will be proved in two steps. First, a new variable will be defined in order to capture the ratio between $\tilde{x}_{k}$ and $\Delta_{k}$, namely $y_{k} \triangleq \tilde{x}_{k} / \Delta_{k}$, and boundedness of that variable will be proved. Secondly, it will be shown that $\Delta_{k}$ asymptotically converges to zero. Consequently, the convergence of $\tilde{x}_{k}$ towards the origin is directly implied. Along the trajectories of (15), the variable $y_{k}$ evolves along the dynamics

$$
y_{k+1}=\frac{1}{\phi_{k}}\left(a y_{k}-\operatorname{sgn}\left(y_{k}\right)\right) .
$$

Fact 1. Trajectories of $y_{k}$ cross the zero axis in finite time.

This means that starting from any initial condition, $\tilde{x}_{0}$ and $\Delta_{0}$, and thus $y_{0}$, there must be a future time $k_{0}<\infty$ such that $y_{k_{0}-1} \cdot y_{k_{0}}<0$. This is easily shown by imagining a trajectory with no zero crossings on $y_{k}$ (hence on $\tilde{x}_{k}$ as $\Delta_{k}$ is always positive). Assuming initially positive $\tilde{x}$, i.e. starting from $y_{0}>0$, we have $y_{k+1}=1 / \lambda^{+}\left(a y_{k}-1\right)$ and hence

$$
y_{k+1}-y_{k}=\frac{1}{\lambda^{+}}\left(a y_{k}-1\right)-y_{k}=\left(\frac{a}{\lambda^{+}}-1\right) y_{k}-\frac{1}{\lambda^{+}}<-\frac{1}{\lambda^{+}}
$$

for the given choice of $\lambda$. Then, starting from $y_{0}>0$, we have $y_{k}<y_{0}-k / \lambda^{+}$and hence there is some constant $k_{0} \leq y_{0} \lambda^{+}$such that $y_{k_{0}-1}>0$ and $y_{k_{0}}<0$. Due to the symmetry of the system equations, a similar argument applies if the trajectory starts from $y_{0}<0$. 
Fact 2. Trajectories of $y_{k}$ are bounded after finite time.

Immediately after a zero crossing of the system (assuming + to - without loss of generality), $y_{k_{0}}$ is bounded as follows

$$
y_{k_{0}}=\frac{1}{\lambda^{+}}\left(a y_{k_{0}-1}-1\right)>-\frac{1}{\lambda^{+}}
$$

hence $\left|y_{k_{0}}\right|<\frac{1}{\lambda^{+}}$, as $y_{k_{0}}<0$. Now if we search for bounds on subsequent samples we must update the growth factor of $\Delta$ to $\lambda^{-}$and compute $y_{k_{0}+1}=1 / \lambda^{-}\left(a y_{k_{0}}+1\right)$, which is positive, as from the bound $0>y_{k_{0}}>-1 / \lambda^{+}$we have

$$
y_{k_{0}+1}>\frac{1}{\lambda^{-}}\left(-\frac{a}{\lambda^{+}}+1\right)>0 .
$$

Moreover, $\left|y_{k_{0}+1}\right|<1 / \lambda^{-}$. These observations are summarized in Fig. 3, where the necessity of two zero crossings after a set of positive values is illustrated, as well as the upper bounds inferred by the switching dynamics. Now as the switching policy is second order, the analysis is concluded by taking one further step. Using $0<y_{k_{0}+1}<1 / \lambda^{-}$, we have $y_{k_{0}+2}=1 / \lambda^{-}\left(a y_{k_{0}+1}-1\right)$, and hence

$$
-\frac{1}{\lambda^{-}}<y_{k_{0}+2}<\frac{1}{\lambda^{-}}\left(\frac{a}{\lambda^{-}}-1\right)
$$

As illustrated in Fig. 3 (left), two situations (a) and (b) are then identified,

(a) $-\frac{1}{\lambda^{-}}<y_{k_{0}+2}<0$. This situation (including the norm bound) is exactly the one found at instant $k_{0}+1$, then it has been already considered

(b) $y_{k_{0}+2}>0$. Then, we have two subsequent positive samples, i.e. the situation of $y_{k_{0}-1}$ is recovered. In that case, the dynamic equation turns into (19) and along it the map $y_{k} \rightarrow y_{k+1}$ is contracting, hence the norm will decrease until a future sign change.

The previous analysis yields that after the first sign change, the state $y_{k}$ remains bounded as

$$
\left|y_{k}\right|<\frac{1}{\lambda^{-}}\left(\frac{a}{\lambda^{-}}-1\right)
$$

With the above facts, the proof of the proposition reduces to show asymptotic convergence to zero of $\Delta_{k}$, and hence concluding convergence of the state $\tilde{x}$. With this objective we will use the following definition,

Definition 2.1: Given a sequence of positive (negative) samples of $y_{k}$, the fly-time is the number of sampling instants elapsed between the two zero-crossings that enclose the signal. For its computation, the first and the last positive (negative) samples are considered. 

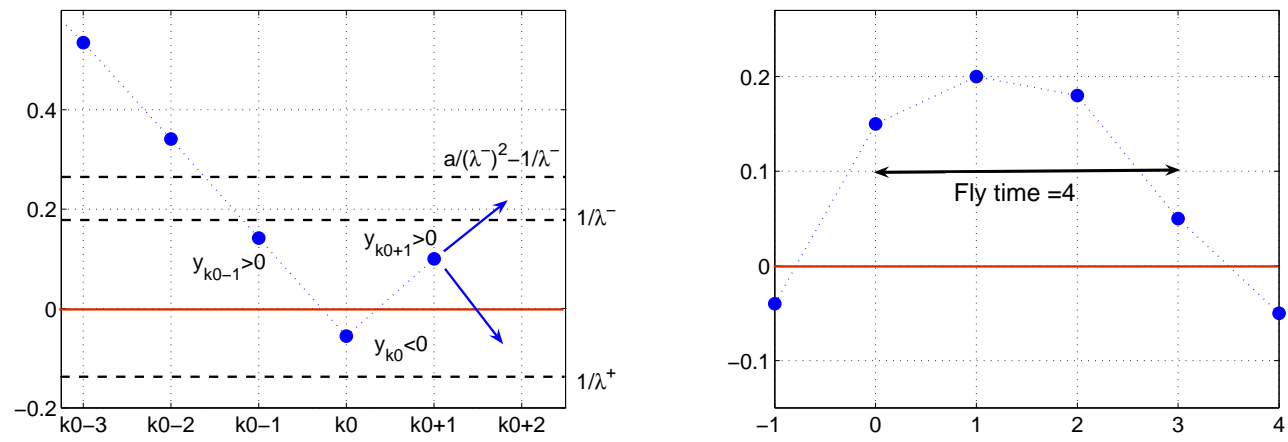

Fig. 3. Left: Behavior of $y_{k}$ at zero crossings. Right: Definition of fly-time.

This magnitude is viewed in Fig. 3 (right). We will compute a non-conservative upper bound on it. Indeed, considering $y_{0}$ as the first positive sample (i.e. resetting the time count), we have along the dynamics (19),

$$
y_{k}=\rho^{k}\left(y_{0}-\frac{1}{a}\left(\frac{\rho^{k}-1}{\rho-1}\right)\right)
$$

then, the zero crossing occurs at the next sampling instant after the time the right hand side of this equation vanishes. Therefore, the fly-time $k^{*}$ is bounded as

$$
k^{*} \leq 1+\log _{\rho}\left(1+y_{0} a(\rho-1)\right) \leq 1+\log _{\rho}\left(1+\frac{a\left(a-\lambda^{-}\right)(\rho-1)}{\left(\lambda^{-}\right)^{2}}\right)=\beta\left(a, \lambda^{-}, \lambda^{+}\right)
$$

for which we have used the finite-time bound on $y_{k}$ (and hence $y_{0}$ ) computed in (20). On the other hand, the duration of a full flying period equals the number of times the $\Delta$ factor is increased as $\Delta_{k+1}=\lambda^{+} \Delta_{k}$, then the net value of $\Delta$ after the flying period, starting from $\Delta_{0}$ is $\Delta_{k}=\left(\lambda^{+}\right)^{k} \Delta_{0}$.

Hence a condition for asymptotic convergence of $\Delta_{k}$ to zero is that, on zero crossings, the net decrease in $\Delta$ compensates the net amount increase over the flying period. This is guaranteed by choosing $\lambda^{-}$such that, (considering $k_{0}$ as the first negative sample, again as in Fig. 3)

$$
\Delta_{k 0+2}=\left(\lambda^{-}\right)^{2} \Delta_{k 0}<\left(\lambda^{-}\right)^{2}\left(\lambda^{+}\right)^{\beta} \Delta_{0}
$$

where the power in $\left(\lambda^{-}\right)^{2}$ has been introduced using the fact that after a flying period, two consecutive zero crossings must occur (see argument of Fact 2). This gives a less restrictive condition on $\lambda^{-}$. Hence, the condition for a net reduction of $\Delta$ after a flying period is

$$
\Delta_{k 0+2}<\Delta_{0} \Leftarrow\left(\lambda^{-}\right)^{2}\left(\lambda^{+}\right)^{\beta}<1
$$


or, equivalently, $\lambda^{-}<\left(\lambda^{+}\right)^{-\frac{\beta}{2}}$ which is the condition (17) stated at the Proposition. From the net convergence of $\Delta_{k}$ to zero and the boundedness of $y_{k}$, we conclude that $\lim _{k \rightarrow \infty} \tilde{x}_{k}=0$. This completes the proof.

In the related literature, some authors have dealt with noisy systems with limited data rates, such as [11]. In that paper, variable length coding is used for stochastic stabilization in the mean square sense. Our approach can also handle the presence of noise in the system dynamics if a minor change in the switching policy is introduced.

Corollary 1: If system (1) is perturbed with a state noise $w_{k}$ such that $\left|w_{k}\right|<W$, as in

$$
x_{k+1}=a x_{k}+b u_{k}+w_{k}
$$

and the same coding-control strategy is applied, with the introduction of a lower bound on $\Delta_{k}$, i.e., substituting the $\Delta$-switching policy by

$$
\Delta_{k+1}=\left\{\begin{array}{l}
\phi_{k+1} \Delta_{k} \text { if } \phi_{k+1} \Delta_{k} \geq \Delta_{\min } \\
\Delta_{\text {min }} \text { otherwise }
\end{array}\right.
$$

where $\Delta_{\min }>W$; then, the error $\tilde{x}_{k}$ is ultimately bounded as

$$
\left|\tilde{x}_{k}\right|<\left(\lambda^{+}\right)^{k *} \Delta_{0} a\left(\lambda^{-}\right)^{-2}\left(1+\frac{W}{\Delta_{\min }}\right)
$$

where

$$
k^{*}=\frac{1}{\log \rho} \log \left(\frac{\lambda^{+} y_{0}}{1-\frac{W}{\Delta_{k}}}\right)
$$

Some details of the proof will be skipped for the sake of space and reported elsewhere. The main argument stems from observing that the evolution of $y_{k}$ is no longer (18), but

$$
y_{k+1}=\frac{1}{\phi_{k}}\left(a y_{k}-\operatorname{sgn}\left(y_{k}\right)+\frac{w_{k}}{\Delta_{k}}\right)
$$

whose solution along a flying period starting at $y_{0}$ (without sign change of $y_{k}$ ) becomes

$$
y_{k}=\left(\frac{a}{\lambda^{+}}\right)^{k} y_{0}+\frac{1}{\lambda^{+}}\left(\sum_{i=0}^{k}\left(\frac{a}{\lambda^{+}}\right)^{i}\right) v_{k-i}
$$

where $v_{k} \triangleq \frac{w_{k}}{\Delta_{k}}-1$. The zero crossings of $y_{k}$ are still guaranteed thanks to the new lower bound on $\Delta_{k}$ and the flying period is limited by $k^{*}$.

Finally, an analysis similar to the noiseless case leads to the bound on $\tilde{x}_{k}$ given in the corollary. Moreover, the closed loop system with nonzero error $\tilde{x}_{k}$ becomes a stable system with a bounded input disturbance perturbation, hence giving a steady state error that is proportional to $W$. 


\section{DATA RATE LIMITS}

Theorem 1 solves the stabilization problem in networked control systems using adaptive Delta Modulation with no restriction on the data rates, apparently in contradiction with the theoretical limits of [13]. In fact, conditions (16)-(17) establish implicitly a constraint between the required data-rate for stabilization and the maximum open-loop eigenvalue of the system.

Actually both parameters are embedded in the constant $a$.

This value, proceeding from the sampling of a continuous system in the form $\dot{x}=\lambda_{\text {cont }} x+$ $B_{\text {cont }} u_{\text {cont }}$, takes the form $a=e^{\lambda_{\text {cont }} T_{s}}$. In a one-bit per sample modulation scheme, which is the case of Delta Modulation we have $a=e^{\lambda_{\text {cont }} / R}$ where $R$ is the data rate in b.p.s. Now, as an upper bound in $a$ will result in an upper bound in the ratio $\lambda_{\text {cont }} / R$, we will investigate what is the maximum value $a_{\max }$ such that for all $a<a_{\max }$ there exist valid choices of parameters $\lambda^{+}$ and $\lambda^{-}$fulfilling (16)-(17).

A detailed numerical analysis of that inequality yields that for $a \leq 1.2$ there are values of $\lambda^{+}$and $\lambda^{-}$that solve the equation. Conversely, for values of $a \geq 1.3226$ no solution has been found numerically. Moreover, as the right hand side of (17) decreases with $a$, there will be no more solutions for greater $a$. An analytical result supporting these facts is presented next.

\section{A. Conditions for solving the parameter tuning equation (17)}

The following proposition states the limits of Theorem 1 in terms of the minimum datarate required for stabilizing a system given its continuous-time open-loop eigenvalues, using Adaptive Delta Modulation. Moreover, as the tuning parameters $\lambda^{-}$and $\lambda^{+}$must be obtained by solving (numerically) the implicit equation (17), finite intervals are provided for scanning those solutions.

Proposition 1: Consider (7) as a result of sampling a continuous-time system $\dot{x}=\lambda_{\text {cont }} x+$ $B_{\text {cont }} u_{\text {cont }}$, with sample time $T_{s}=1 / R$ and Adaptive Delta Modulation one-bit state coding as (14). Then, a sufficient (possibly conservative) condition for closed-loop stability with feedback $u=K \hat{x}$ where $\hat{x}$ is the output of the decoder is $R>\lambda_{\text {cont }} / \log (1.3226)$ bits per second. Moreover, a less conservative search for the tuning parameters $\lambda^{+}$and $\lambda^{-}$satisfying (17) is to be carried out numerically scanning the finite interval

$$
\left.\lambda^{-} \in(0,1)\right] \times\left[\lambda^{+} \in\left(a, a \frac{1+\sqrt{1+\frac{4}{a(a-1)}}}{2}\right)\right]
$$


Proof: (Sufficient condition) We are concerned with the existence of solutions satisfying inequality (17), i.e. $\lambda^{-}<\left(\lambda^{+}\right)^{-\frac{\beta}{2}}$ within the range of validity of the constants, i.e. $\left[\lambda^{-} \in\right.$ $(0,1)] \times\left[\lambda^{+} \in(a, \infty)\right]$. Using the property $\lambda^{-}=\left(\lambda^{+}\right)^{\left(\frac{\log \lambda^{-}}{\log \lambda^{+}}\right)}$, we can rewrite (17) as

$$
\frac{\log \lambda^{-}}{\log \lambda^{+}}<-\frac{1}{2}\left(1+\frac{\log \left(1+\frac{a\left(a-\lambda^{-}\right)(\rho-1)}{\left(\lambda^{-}\right)^{2}}\right)}{\log (\rho)}\right) .
$$

Now a sufficient condition for its solution is obtained by taking $\operatorname{limit}^{1}$ as $\rho \rightarrow 1^{+}$, which gives

$$
\left.\log \lambda^{-}<-\frac{\log a}{2}\left(1+\frac{a\left(a-\lambda^{-}\right)}{\left(\lambda^{-}\right)^{2}}\right), \Leftrightarrow\left(\lambda^{-}\right)^{2} \log \lambda^{-}<-\frac{\log a}{2}\left(\left(\lambda^{-}\right)^{2}+a^{2}-a \lambda^{-}\right)\right) .
$$

The left hand side of the last expression is minimized to -0.1839 at $\lambda^{-}=0.6070$. Substituting this value in the right hand side, we have the inequality $-0.183<-(\log a) / 2\left(a^{2}-0.6070 a+0.6070^{2}\right)$ that is fulfilled by values of $a$ below 1.3226. This means that if $a$ is below that value, then we can find tuning parameters $\lambda^{-}=0.6070$ and $\lambda^{+}$close enough to $a$ (from the limit as $\rho \rightarrow 1^{+}$) such that (17) holds and the coding scheme can be implemented. Now from the relation between the discrete and continuous-time formulation, the relation $R>\lambda_{\text {cont }} / \log (1.3226)$ is implied.

(Parameter search interval) If the sufficient condition on the data rate (for which the parameter tuning procedure is given above) is not satisfied, (17) might be solved numerically. To this end, a finite interval for the tuning parameters will be obtained by analyzing necessary conditions for (17), also rewritten as

$$
\log \lambda^{-}<-\frac{1}{2} \frac{\log \lambda^{+}}{\log (\rho)}\left(\log (\rho)+\log \left(1+\frac{a\left(a-\lambda^{-}\right)(\rho-1)}{\left(\lambda^{-}\right)^{2}}\right)\right),
$$

for which, as $\lambda^{+}>\rho$, it is necessary that

$$
\log \lambda^{-}<-\frac{1}{2}\left(\log (\rho)+\log \left(1+\frac{a\left(a-\lambda^{-}\right)(\rho-1)}{\left(\lambda^{-}\right)^{2}}\right)\right),
$$

and the right hand side of this expression is upper bounded by

$$
\text { r.h.s. of }(25)<-\frac{1}{2}\left(\log (\rho)+\log \left(\frac{a(a-1)(\rho-1)}{\left(\lambda^{-}\right)^{2}}\right)\right)=-\frac{1}{2} \log \left(\rho\left(\frac{a(a-1)(\rho-1)}{\left(\lambda^{-}\right)^{2}}\right)\right) \text {. }
$$

Hence the necessary condition for the existence of solutions is

$$
\left(\lambda^{-}\right)^{2}<\left(\rho\left(\frac{a(a-1)(\rho-1)}{\left(\lambda^{-}\right)^{2}}\right)\right)^{-1}
$$

\footnotetext{
${ }^{1}$ Intuitively, the best choice for $\lambda^{+}$is to be close to $a$, as this would reduce the flying period and impose a less conservative constraint on $\lambda^{-}$for a net reduction of $\Delta_{k}$.
} 
i.e. $\rho a(a-1)(\rho-1)<1$ and hence $\rho(\rho-1)<1 /(a(a-1))$. Therefore, the valid choices of $\rho$ are such that

$$
\frac{1-\sqrt{1+\frac{4}{a(a-1)}}}{2}<\rho<\frac{1+\sqrt{1+\frac{4}{a(a-1)}}}{2} .
$$

But, as $\lambda^{+}>a$, the search interval becomes (24).

\section{RECOVERY AND PERSISTENCE ISSUES}

In previous papers presented in this field, such as [14] and [7] an explicit bound on the initial state $x\left(t_{0}\right)$ is required in order to initialize the encoder. The proposed bypass is to execute an initial uncontrolled stage (with undesired transient effects) to estimate that bound (See [7], Section III), but it is not stated how to switch back and forth between stages in case a disturbance drives away the state (it is a known fact that the switching policy is crucial in stability analysis). On the other hand, [15] proposes a zoom-out and then zoom-in mechanism (in that particular ordering), and it deals only with continuous-time quantization (and hence unlimited data rate).

Our claim is that our algorithm does not distinguish between different stages, i.e. the mathematical definition is unique, and it does not require any bound on the initial state. As a consequence, the algorithm allows the state to escape momentarily at any time due to unattended unbounded finite-time disturbances, and after them it will recover stability.

It must be acknowledged, however, that a temporary loss of communication could have a side effect: the equi-memory property may be lost, i.e. $\Delta_{k}$ may differ between receiver and transmitter. In that case, a recovery procedure for synchronizing both ends is proposed as follows. Considering $k^{*}$ as the maximum flying period (see proof of 1), we propose the following resynchronization algorithm:

\section{Initialization Sequence}

\section{Coding operation}

1) $N_{s}=0, x_{0}=0, \Delta_{0}=\Delta^{*} \quad$ 1) If $\delta_{k}=\delta_{k-1}$ set $N_{s}=N_{s}+1 ; \quad$ else set $N_{s}=0$

2) Wait until $\delta_{k} \neq \delta_{k-1}$

2) If $N_{s}=k^{*}+1$ go to Initialization Seq.

3) Go to Coding operation

3) Apply (8)-(10) (enc.), or (11)-(13) and (14) (dec.)

Here, $\Delta^{*}>0$ is the freely-chosen (but shared) initial value of the quantization step. Thanks to the global stability of the system with unconstrained encoder initialization, this algorithm at both the emitter and receiver guarantees that, in the case of temporary loss of communication provokes instability of the error dynamics (loss of synchronization, or equi-memory), both communication 

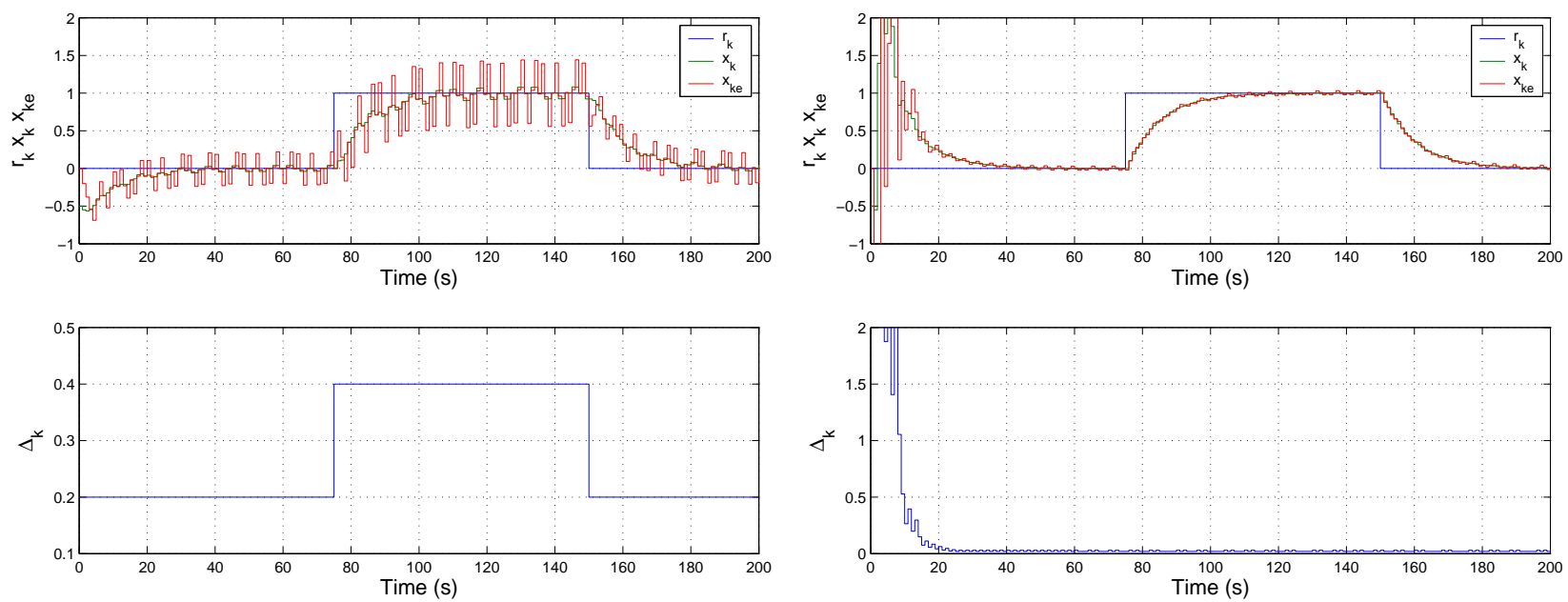

Fig. 4. System (7)-(14) has been simulated for the set of values $a=1.1, b=1, K=0.2, x_{k}(0)=-0.5, \hat{x}_{k}(0)=0$, with $\Delta_{0}=5, \lambda^{-}=0.4, \lambda^{+}=1.21$, according to conditions (16) and (17). The simulation compares the fixed-gain $\Delta$ coding scheme (left figure) [2], with the proposed adaptive scheme (right figure). In the first case, the value of $\Delta$ is changed at pre-specified time instants whereas the adaptive law for $\Delta$ allows for better granularity which is adapted as a function of the closed-loop error signal improving the system performance while enlarging the domain of attraction.

ends will reset their Delta Modulators and recover stability after a transient. The validity of this procedure relies in the fact that, in normal operation, no more than $k^{*}$ consecutive samples with the same value of $\delta_{k}$ can occur. Step 3 in the initialization sequence is required because the flying period is bounded only after the first zero crossing the error signal.

\section{Simulations}

Simulations are illustrated in Fig. 4. The upper plots show $x_{k}, \hat{x}$ and $r_{k}$. The lower figures show the time evolution of $\Delta_{k}$ for both; fixed and adaptive cases. As expected, the adaptive law performs over the fixe case as the tracking error decrease up to an arbitrarily small value dictate by the minimum saturation for $\Delta_{\min }=0.05$. Without this saturation, $\Delta_{k}$ would decrease to zero indefinitely annulling the system capacity to recover from future perturbations.

\section{CONCLUSIONS}

In this paper we have investigated the stability properties of the Delta-modulation coding rule, when used as a transmission means in networked controlled linear systems. It was first shown that the standard form of the $\Delta-M$ algorithm can be modified, including information about the 
system and the controller. These results were extended to the case of adaptive $\Delta_{k}$. An explicit adaptation rule was proposed and the range of parameters were derived to ensure asymptotic stability. These results displayed a limit on the maximum unstable eigenvalues of the system that are compatible with the ones given in [13].

\section{REFERENCES}

[1] Proakis J.-G, Digital Communications. McGraw-Hill, Inc. Series in electrical and computer enginering.

[2] C. Canudas-de-Wit, F. Rubio, J. Fornes, and F. Gomez-Estern, "Differential coding in networked controlled linear systems," American Control Conference. Silver Anniversary ACC. Minneapolis, Minnesota USA, June 2006.

[3] C. A. I. Lopez and C. Canudas-de-Wit, “Compensation schemes for a delta-modulation-based ncs," ECC'07 USA, 2007.

[4] C. Canudas-de-Wit, J.Jaglin, and C. Siclet, "Energy-aware 3-level coding and control co-design for sensor network systems," in Conference on Control Application, Singapore, 2007.

[5] Ishii H. and T. Başar, "Remote control of lti systems over networks with state quatization," System and Control Letters, no. 54, pp. 15-31, 2005.

[6] Elia N. and S.-K. Mitter, "Stabilization of linear systems with limited information," IEEE Transaction on Automatic Control, vol. 46, no. 9, pp. 1384-1400, September 2001.

[7] Liberzon D., "On stabilization of linear systems with limited information," IEEE Transaction on Automatic Control, vol. 48, no. 2, pp. 304-307, February 2003.

[8] Lemmon M. and Q. Ling, "Control system performance under dynamic quatization: the scalar case," in 43rd IEEE Conference on Decision and Control, Atlantis, Paradice Island, Bahamas, 2004, pp. 1884-1888.

[9] Tan S., X. Wei, and J.-S. Baras, "Numerical study of joint quatization and control under block-coding," in 43rd IEEE Conference on Decision and Control, Atlantis, Paradice Island, Bahamas, 2004, pp. 4515-4520.

[10] Li K. and J. Baillieul, "Robust quatization for diginal finite communication bandwidth (dfcb) control," IEEE Transaction on Automatic Control, vol. 49, no. 9, pp. 1573-1584, September 2004.

[11] Girish N. Nair and Robin J. Evans, "Stabilizability of stochastic linear systems with finite feedback data rates," SIAM J. Control Optim., vol. 43, no. 2, pp. 413-436, 2004.

[12] C. Canudas-de-Wit, J.Jaglin, and C. Siclet, "Delta modulation for multivariable centralized linear networked controlled systems," in 47th Conference on Decision and Control, Cancun, Mexico, 2008.

[13] S. Tatikonda and S. Mitter, "Control under communication constraints," IEEE Transaction on Automatic Control, vol. 49, no. 7, pp. 1056-1068, July 2004.

[14] Hespanha J.-P., Ortega A., and Vasudevan L., "Towards the control of linear systems with minimum bit-rate," in 15th Int. Symp. Mathematical Theory of Networks and Systems (MTNS), Notre Dame, IL, USA, 2002.

[15] Brockett R.-W. and Liberzon D., "Quantized feedback stabilization of linear systems," IEEE Transactions on Automatic Control, vol. 45, no. 7, pp. 1279-1289, July 2000. 